https://doi.org/10.23913/ride.v10i19.524

Artículos Científicos

\title{
Transversalización curricular ambiental en educación superior mediante comités de diseño curricular
}

\section{Environmental Curricular Mainstreaming Strategy in Higher Education Through Curricular Design Committees}

\begin{abstract}
Transversalização dos currículos ambientais no ensino superior por meio de comitês de design de currículo

José Luis Aparicio López

Universidad Autónoma de Guerrero, México

joselopez@uagro.mx https://orcid.org/0000-0002-4586-6954

Columba Rodríguez Alviso

Universidad Autónoma de Guerrero, México columbaalviso@uagro.mx https://orcid.org/0000-0001-9600-8776
\end{abstract}

\section{Resumen}

La problemática ambiental es un desafío para las instituciones educativas que debe afrontarse desde todas sus funciones, y con especial énfasis desde la docencia y la investigación. Este trabajo expone la experiencia de la conformación de comités de diseño curricular como una estrategia para desarrollar la transversalización curricular del eje medio ambiente en los planes de estudio de educación superior en la Universidad Autónoma de Guerrero, México. A través del método de investigación-acción, se trabajó de manera colaborativa con comités de 15 programas educativos en dos ciclos (2012-2013 y 2016-2017). Como resultado, se presenta una estrategia dividida en cuatro etapas: $a$ ) conformación del comité; $b$ ) diagnóstico de la presencia del medio ambiente en el plan de estudios; $c$ ) transversalización curricular del eje medio ambiente, y $d$ ) definición del perfil de egreso y elaboración del programa de unidad de aprendizaje. La 


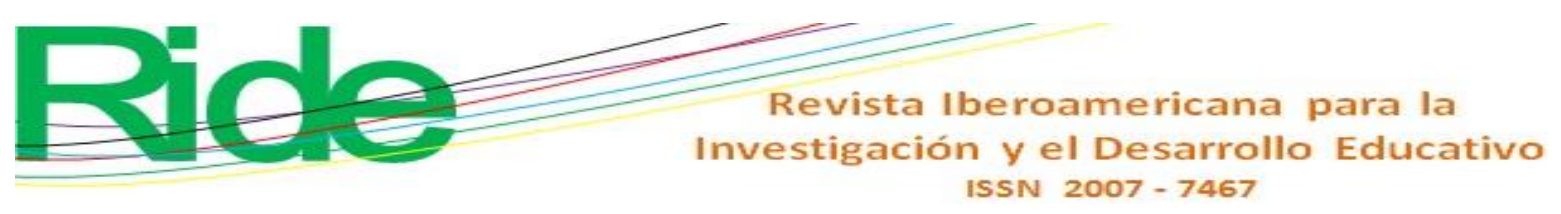

estrategia se acompaña de dos instrumentos para su implementación. Se concluye en la necesidad de darle continuidad a la propuesta, fortalecerla y generalizarla en los demás programas educativos de educación superior de la universidad, así como iniciar un nuevo ciclo de investigación.

Palabras clave: diseño curricular, educación superior, investigación-acción, medio ambiente, transversalización curricular.

\section{Abstract}

The environmental problem represents a challenge for educational institutions, which must be addressed from all its functions, especially in teaching and research. In this work it is presented the experience of the conformation of curriculum design committees as a strategy to develop the curricular mainstreaming of the environment axis in higher education study plans at the Autonomous University of Guerrero, Mexico. Through the action research method, collaborative work was carried out with committees of 15 educational programs in two cycles (2012-2013 and 2016-2017). As a result, a strategy divided into four stages is proposed: a) conformation of the committee; b) diagnosis of the presence of the environment in the curriculum; c) curricular mainstreaming of the environment axis; and d) definition of the graduate profile and development of the learning unit program. Two instruments for its implementation accompany the strategy. It concludes in the need to give continuity to the proposal, strengthen it and generalize it in the other higher education educational programs of the university, as well as start a new research cycle.

Keywords: curricular design, higher education, research-action, environment, curricular mainstreaming. 


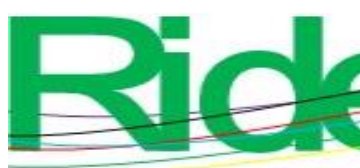

Revista Iberoamericana para la Investigación y el Desarrollo Educativo

ISSN $2007-7467$

2012, esta comisión consideró importante sistematizar los trabajos académicos e involucrar a los docentes como actores principales del proceso educativo; así, se decidió organizarlos mediante la figura de comités de diseño curricular (CDC). Una primera tarea consistió en la incorporación en el currículo de los temas emergentes sociales indicados en el Modelo Educativo y Académico de la Universidad Autónoma de Guerrero (UAGro, 2004), entre los que estaban: derechos humanos, equidad, multiculturalidad y medio ambiente; si bien estaban señalados, se carecía de orientaciones metodológicas para su implementación. Uno de los temas que por su naturaleza generó mayor interés fue el de medio ambiente y desarrollo sustentable. El proceso para la incorporación de estos temas emergentes sociales fue posible a partir de diversos eventos académicos que fortalecieron las competencias del docente, como fueron: Primer Diplomado en Competencias Docentes 2012: Educación, Ciudadanía y Derechos Humanos; Primera Semana de Actualización Docente 2013; Segunda Etapa de Actualización Docente 2014; Actualización Docente Verano 2015, y Actualización Docente Verano 2016. Por su importancia y trascendencia, se destaca el Primer Encuentro de Comités de Diseño Curricular en la Implementación de Planes de Estudio por Competencias, llevado a cabo en octubre de 2014, donde los participantes pudieron intercambiar experiencias y evaluar su desempeño (UAGro, 2017).

\section{Antecedentes}

Las instituciones de educación superior deben experimentar cambios profundos en sus estructuras administrativas y en su quehacer docente para estar a la altura de los retos y exigencias de una sociedad cada vez más demandante. Dentro de estas instituciones formadoras de profesionistas, la universidad cobra mayor relevancia, pues es depositaria no tan solo de mayores expectativas, sino de presiones políticas y sociales para cumplir dicha función (Aparicio, 2014). Se demandan procesos renovadores que permitan superar la tradición de currículos centrados en las disciplinas más que en los valores (González y Arias, 2015). Las instituciones educativas, además de enfocarse en los temas disciplinares, deben atender las temáticas consideradas emergentes sociales, como la ambiental. No podemos continuar con procesos educativos fundamentados en modelos pedagógicos de hace 50, 100 o 200 años (Clavijo, 2018).

En la literatura revisada se encontraron experiencias que pueden clasificarse en dos vertientes: las que incorporan el medio ambiente como un todo integrado, y aquellas que lo abordan desde la sustentabilidad o el desarrollo sustentable. En este trabajo se consideró a la 


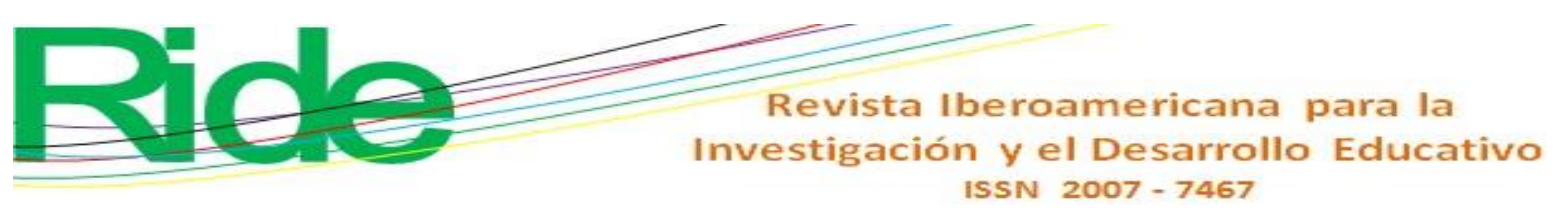

sustentabilidad y el desarrollo sustentable en tanto que contemplan aspectos ambientales, sociales y económicos (Lozano, 2008).

Mora (2012) realizó un estudio para diagnosticar las concepciones del profesorado sobre la incorporación de la dimensión ambiental en el currículo de la Facultad de Medio Ambiente, en la Universidad Distrital en Bogotá, Colombia. El resultado evidenció una incipiente ambientalización curricular, producto de una formación monodisciplinar; fue necesario fortalecer aspectos pedagógico-didácticos, así como la constitución de un comité curricular que trabajara la inclusión ambiental en los contenidos y la mejora de las prácticas educativas en el aula.

Cárdenas (2013) delineó una propuesta para introducir en las universidades peruanas la dimensión ambiental, con énfasis en la responsabilidad social. Refirió la necesidad de integrar un organismo que propusiera y ejecutara las acciones que la misma universidad definiera. Esta figura, llamada Comité Ambiental, tenía como objetivo la promoción, gestión, coordinación y supervisión de los procesos de incorporación ambiental en las funciones sustantivas (docencia, investigación, extensión y gestión). Por su parte, Espejel, Castillo y Martínez (2011) propusieron un modelo de educación ambiental para el nivel medio superior de la región Puebla-Tlaxcala, México; también concluyeron en la importancia de integrar un comité ambiental, conformado por docentes, directivos, administrativos e instituciones ambientales, con el objetivo de lograr la implementación de las tareas programadas.

Otra experiencia fue la de Comisión Mexicana de Cooperación-Organización de las Naciones Unidas para la Educación, la Ciencia y la Cultura (Conalmex-Unesco), con acciones pedagógicas orientadas a la incorporación ambiental en el currículo en el estado de Tabasco a través de un comité regional, cuyo enfoque fue tomar como actor principal al docente, y dotarlo de materiales, capacitación y seguimiento (Arias y Ramírez, 2006).

Stubbs y Cocklin (2008) puntualizaron que en el desarrollo de estos procesos de cambio es importante que los estudiantes conozcan los conceptos de ambiente y sustentabilidad, y los integren en su vida profesional; así también, que sean considerados en la capacitación, puesto que el docente aparece como responsable último del eventual éxito de las innovaciones curriculares, al llevar el cambio didáctico al aula. La Hogeschool-Universiteit Brussel diseñó un manual y un método para la integración del desarrollo sustentable en sus planes de estudio; se centró en las carreras de ingeniería comercial a nivel licenciatura y maestría; el propósito fue motivar a los maestros para integrar este tema en sus cursos y desarrollarlo con los estudiantes (Ceulemans y De Prins, 2009). 


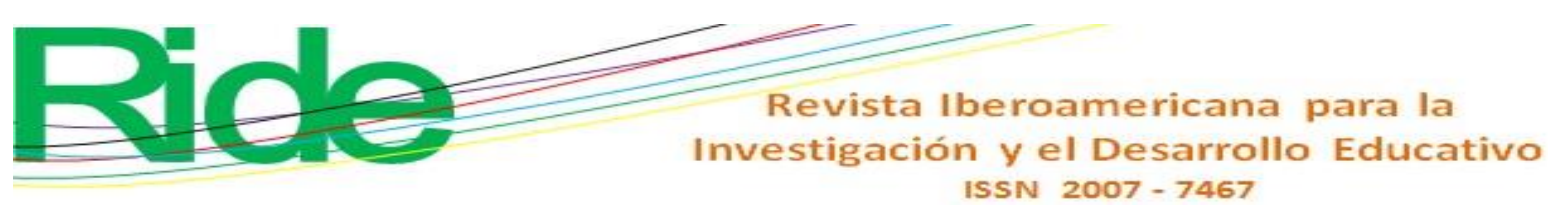

Las experiencias hasta aquí comentadas dan cuenta de los esfuerzos institucionales por lograr la incorporación de los temas emergentes en el currículo (como el ambiental); también coinciden en la importancia de contar con un grupo, comisión o comité curricular que, a través de la estrategia del trabajo colaborativo de pequeños grupos, con la misma información y objetivos (Moreno, 2011), logre involucrar al docente como el principal generador de información, ya que es él y sus estudiantes en el aula quienes conforman un verdadero ecosistema donde se aplican y desarrollan las propuestas formativas previamente diseñadas (Domínguez, Medina y Sánchez, 2011).

\section{Fundamentación teórica}

La educación es un medio para mitigar los problemas ambientales globales. Por ello, es necesario delimitar conceptualmente qué es el ambiente. Los términos medio y ambiente son redundantes, pero debido a su uso reiterado y constante se ha acuñado la expresión medio ambiente. Así, el uso de medio, ambiente o medio ambiente se refiere al entorno en que se desenvuelven los seres humanos (Programa de Naciones Unidas para el Medio Ambiente [PNUMA], 2006). El ambiente hace referencia a la "naturaleza transformada por la actividad humana, (...) no es solo lo que 'rodea' sino también un producto de 'aquello que es rodeado"' (Bocco y Urquijo, 2013, p. 84). Es un objeto híbrido que engloba todas las relaciones entre naturaleza y sociedad, entre naturaleza y cultura (Galochet, 2009). Al ser un concepto inter y multidisciplinario, tiene variadas acepciones: preservación ambiental, efectos ambientales del cambio climático, o como uno de los componentes del desarrollo sustentable: ambiental, económico y social (González, 2000; Giannuzzo, 2010). Aquí se adopta la acepción de preservación, pero no reducida a lo ecológico, sino a una problemática contenida en la relación de naturaleza y sociedad que debe ser abordada desde el ámbito educativo.

Al ser global y de efectos devastadores, la problemática ambiental debe estudiarse de manera transversal en todas las asignaturas de todos los niveles educativos. Con esta disposición, la transversalización se presenta como una estrategia curricular pertinente, en función de establecer ejes o temas considerados prioritarios para la formación integral de los estudiantes, en todos los programas, actividades y planes de estudio contemplados en el proyecto institucional (Velásquez, 2009). Implica, entre otros procesos, el rediseño curricular, desde el estudio de las disciplinas y sus campos ocupacionales, y la redefinición de los perfiles profesionales 


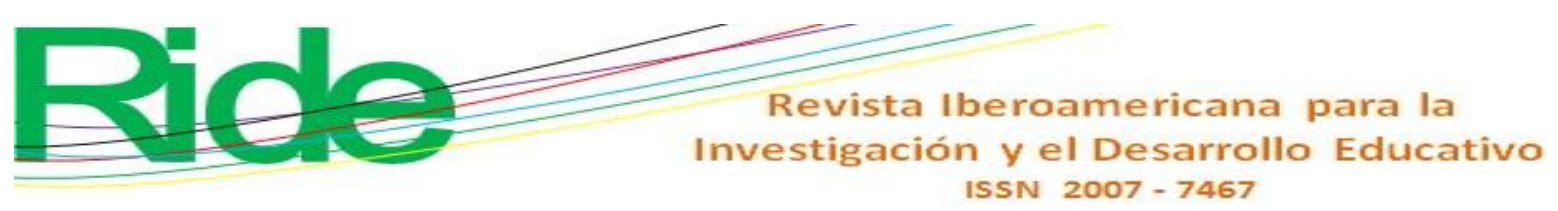

"ambientalizados", así como los planes de estudio y las unidades de aprendizaje (Santamaría y Bravo, 2015).

Es pertinente hacer una distinción entre temas y ejes. Los temas transversales son contenidos educativos no disciplinares, conocidos también como emergentes sociales: salud, consumo o medio ambiente; mientras que los ejes o líneas transversales tienen el potencial de estructurar esos emergentes sociales que se "impregnan" en el currículo (Yus, 1996). En este caso, por tratarse de diseño curricular, se aludieron como ejes transversales.

La transversalización de los planes de estudios puede darse en dos sentidos: horizontal, entretejida en diferentes cursos, y vertical, a través de un curso de desarrollo sustentable dentro del currículo (Ceulemans y De Prins, 2009). Lozano (2008) plantea cuatro enfoques: $a$ ) inclusión del tema y materiales ambientales en un curso o módulo, $b$ ) inclusión de un curso específico, $c$ ) entrelazar el concepto de desarrollo sustentable en los cursos disciplinares regulares y $d$ ) el desarrollo sustentable como especialidad; el autor especifica que estos enfoques se usan de forma independiente; otros autores los combinan. Para Fernández (2003), la transversalidad debe establecer puentes de unión entre el saber académico (aprender a aprender) y el saber vital o vulgar (aprender a vivir); implica la toma de decisiones curriculares, por lo que es preciso que se realice a través de la discusión y reflexión colegiada; los comités de diseño se presentan como la mejor opción, pues permiten el desarrollo, cambio, mejora, adecuación, crecimiento y participación del propio docente en un contexto de libre participación.

El diseño curricular es "un proceso de toma de decisiones para la elaboración o ajuste del currículo" (Tovar y Sarmiento, 2011, p. 509); da respuesta no solo a los problemas de índole educativa, sino también a los de carácter económico, político y social. "Comprende las mismas etapas de la planeación (diagnóstico, análisis de la naturaleza del problema, diseño y evaluación de las posibilidades de acción, e implantación y evaluación" (Díaz, Lule, Rojas y Saád 1990, p. 22).

En ese contexto de rediseño curricular, en 2011 se constituyeron en la UAGro los primeros CDC para la actualización de los planes de estudios de educación superior, complementados con un proceso de capacitación que les permitiera pasar de un modelo tradicional por objetivos a uno por competencias (Aparicio, Torres, Sánchez y Gutiérrez, 2015).

Este trabajo tuvo como objetivo compartir la experiencia sobre una estrategia educativa para la transversalización ambiental en los planes de estudio de educación superior en la UAGro, México, mediante la constitución de CDC. 


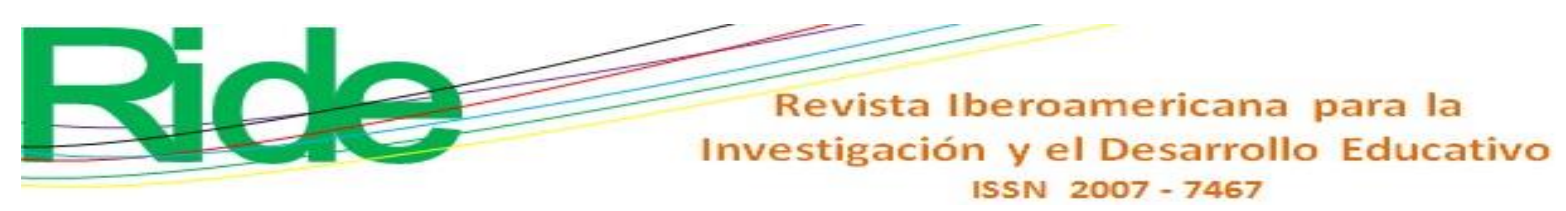

Metodología

Esta investigación es cualitativa, con fundamento en la investigación-acción; es empírica; recupera las ideas y propuestas, basadas en la experiencia, de los docentes participantes.

El trabajo implicó la colaboración de 15 CDC de igual número de programas educativos; 14 reformados en 2011 y 1 creado en 2016 (Ciencias y Tecnologías de la Información). En apego a la metodología de la investigación-acción (Latorre, 2003), se desarrollaron dos ciclos, tal y como se muestra en la tabla 1.

Tabla 1. CDC participantes

\begin{tabular}{|l|l|l|}
\hline \multicolumn{1}{|c|}{ Ciclo } & Periodo & \multicolumn{1}{|c|}{ Programas Educativos } \\
\hline Primero & $2012-$ & $\begin{array}{l}\text { 1) Biólogo, 2) Ingeniería en Sistemas Ambientales, 3) Ingeniería } \\
\text { en Producción Sustentable, 4) Ingeniería en Recursos Maderables } \\
\text { y no Maderables, 5) Ingeniero Topógrafo y Geomático, 6) } \\
\text { Licenciado en Enfermería (Taxco), 7) Licenciado en Enfermería } \\
\text { (Chilpancingo), 8) Licenciado en Artes, 9) Licenciado en Ciencias } \\
\text { de la Educación, 10) Licenciado en Ciencia Política y } \\
\text { Administración Pública, 11) Licenciado en Sociología de la } \\
\text { Comunicación y Educación, 12) Licenciado en Desarrollo } \\
\text { Regional }\end{array}$ \\
\hline Segundo & $2016-$ & $\begin{array}{l}\text { 1) Ciencias y Tecnologías de la Información, 2) Licenciado en } \\
\text { Contaduría, 3) Licenciado en Administración de Empresas }\end{array}$ \\
\hline
\end{tabular}

Fuente: Elaboración propia

Al iniciar el estudio, en 2012, ya estaban conformados los comités participantes, cada uno constituido por un mínimo de cinco y un máximo de nueve docentes, incluyendo un coordinador encargado de estructurar y gestionar el programa de actividades acordado. El proceso para la transversalización se construyó de manera alterna a un proceso de diseño y actualización curricular en la UAGro, con fundamento en la metodología del libro Diseño, actualización y evaluación de planes de estudio de nivel Licenciatura (UAGro, 2012). De esta metodología institucional se retomaron elementos para llegar a la transversalización, como son: redefinición del perfil de egreso, construcción de competencias y elaboración del programa de unidad de aprendizaje. 


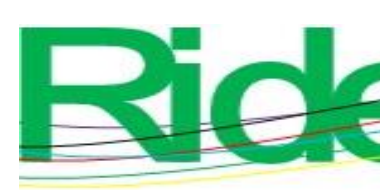

Revista Iberoamericana para la
Investigación y el Desarrollo Educativo
ISSN $2007-7467$

- Reflexión. Se analizaron los resultados obtenidos en la actuación y en la observación; se realizaron ajustes para dar más claridad y coherencia a los instrumentos y se hicieron algunas adecuaciones al contenido.

\section{Segundo ciclo}

- $\quad$ Planificación. En 2016 se planificó un segundo ciclo investigativo y se diseñó un proceso de capacitación para el fortalecimiento de las competencias docentes, en las temáticas: Educación ambiental para la sustentabilidad, Transversalidad del eje medio ambiente y Elaboración de secuencia didáctica.

- Actuación. En 2017 se desarrolló, por separado, el proceso de capacitación; primero al CDC del programa de Ciencias y Tecnologías de la Información; posteriormente a los de Contaduría y Administración de Empresas; el abordaje de los temas estuvo a cargo del comité de expertos en diseño curricular y transversalidad, con una duración de seis horas cada uno.

- Observación. Se identificó el grado de avance en el dominio de los conceptos relacionados con el diseño curricular (perfil de egreso, competencias y programa de unidad de aprendizaje), las temáticas ambientales y la estrategia de la transversalidad.

- Reflexión. Los resultados obtenidos en el primero y segundo ciclo son el fundamento para la presentación de la "Metodología para la transversalización del eje medio ambiente" en el siguiente apartado.

\section{Resultados}

Los hallazgos se dividen en tres partes. En primer lugar, se presentan las condiciones iniciales de la investigación; se continúa con los cambios mostrados por los CDC para la transversalización del eje medio ambiente; la tercera parte se deriva de la reflexión de los docentes y expone la estrategia de los autores para iniciar un nuevo ciclo.

\section{¿Cómo se inició el trabajo con los CDC?}

Al inicio de ambos ciclos los docentes de los 15 CDC manifestaban desconocimiento o una falsa conceptualización sobre los problemas sociales emergentes (dentro de ellos el medio ambiente) y la transversalidad como estrategia curricular. 


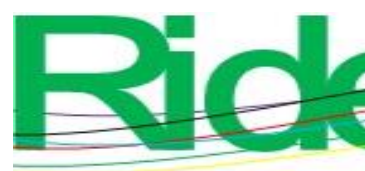

Revista Iberoamericana para la Investigación y el Desarrollo Educativo ISSN $2007-7467$

Además, evidenciaron desconocimiento del plan de estudios y los programas de cada unidad de aprendizaje, a causa de una falta de aplicación de la normativa institucional, pues la asignación de la carga académica se realiza de acuerdo con la categoría laboral y no el dominio disciplinar; no siempre se respeta el perfil del docente.

\section{¿Qué cambios se generaron para la transversalización del eje medio ambiente?}

El proceso de capacitación en los temas de diseño curricular, educación ambiental y transversalidad en el segundo ciclo permitió realizar ajustes a la estructura de los instrumentos utilizados, así como la adecuación y clarificación de los componentes del medio ambiente.

Con observaciones de los tres CDC participantes en el segundo ciclo, y con el apoyo del comité asesor, se hicieron dos cambios en los instrumentos 1 y 2, retomados de la propuesta de Aparicio, Rodríguez y Beltrán (2014); originalmente había un verbo para los cuatro conocimientos (construye): en el segundo se hizo una diferenciación (conoce, identifica, distingue y relaciona); asimismo, en el componente de los conocimientos se incluyó la interrelación del aire, agua, suelo y ecosistemas en la biodiversidad, más acorde con la conceptualización adoptada sobre medio ambiente (ver Anexos).

Otro aspecto fue fundamentar en la EBC el programa y la secuencia didáctica para el curso- taller, lo que permitió a los docentes tener más referencias conceptuales, procedimentales y actitudinales para la incorporación del medio ambiente en su plan de estudios y unidades de aprendizaje.

Se pudo establecer que el trabajo colegiado de los docentes, mediante la constitución de los CDC, es compatible y pertinente con la estrategia para la transversalización del eje medio ambiente en los planes de estudio de licenciatura.

Con el comité de expertos y los coordinadores de los CDC, se documentó cada una de las fases y los ciclos desarrollados. Para una mayor comprensión, se sistematizó la información y se presenta a manera de metodología que permita darle continuidad con un nuevo proceso investigativo, en la búsqueda de institucionalizarla en la universidad.

Uno de los logros más significativos fue la reconceptualización sobre la problemática ambiental y el trabajo colegiado, lo que facilitó en desarrollo de los trabajos sobre la transversalización. 


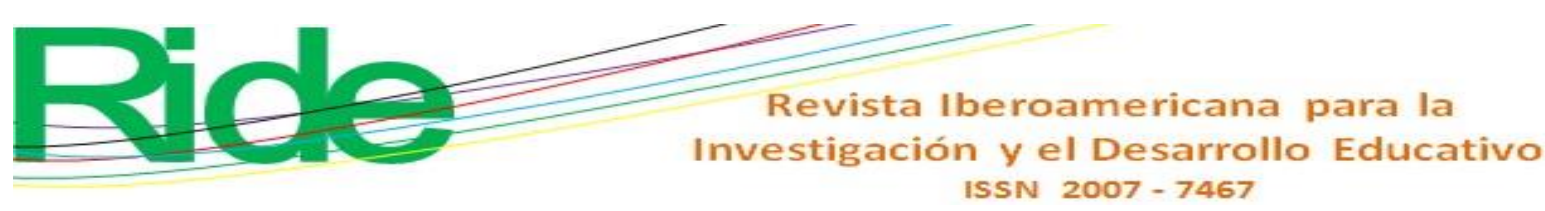

Se aspira a que los resultados hasta ahora obtenidos sean la base para la planeación de un tercer ciclo de intervención, que conlleve a lograr la impregnación del eje medio ambiente en todo el plan de estudios, desde el perfil de egreso, las competencias a desarrollar en cada programa de asignatura, hasta la secuencia didáctica o plan de clase, y lograr que la transversalidad sea una realidad en el aula o salón, coadyuvante con los procesos formativos integrales.

\section{Estrategia para transversalizar}

1. Conformación del CDC:

a) El proceso pertenece a la educación formal, por lo que se debe institucionalizar mediante una carta compromiso, signada entre la Dirección General de Educación Media Superior y Superior, el director de la escuela y el coordinador del CDC, donde se manifieste explícitamente el interés por reformar su plan de estudios. Este proceso es acompañado por un grupo de expertos en transversalidad, acreditados por la institución educativa.

b) Se constituye o ratifica el $\mathrm{CDC}$, integrado por docentes del propio programa educativo participante que muestren disposición para el trabajo colaborativo, así como conocimientos en el diseño curricular por competencias.

c) Se diseña un proceso de intervención mediante tres líneas de capacitación y formación: educación ambiental para la sustentabilidad, transversalidad del eje medio ambiente y diseño curricular (perfil de egreso, competencias docentes, programa académico y secuencia didáctica).

d) Se trabaja con los docentes participantes en la socialización y manejo de los instrumentos 1 y 2. Estos son el resultado de la reflexión colectiva en la investigación-acción, por lo que son idóneos para generalizarse en cualquier programa educativo de nivel superior de la UAGro.

e) Se estructura un cronograma de trabajo para el proceso de actualización de cada programa bajo la conducción de un coordinador.

2. Diagnóstico de la presencia del medio ambiente en el plan de estudios:

a) Se aplica el instrumento 1 para el diagnóstico y valoración del nivel de presencia del eje medio ambiente (conformado por conocimientos, habilidades, actitudes y valores) en el programa educativo o unidad de aprendizaje; se aplica a cada coordinador del programa educativo, así como a los docentes integrantes del comité. 


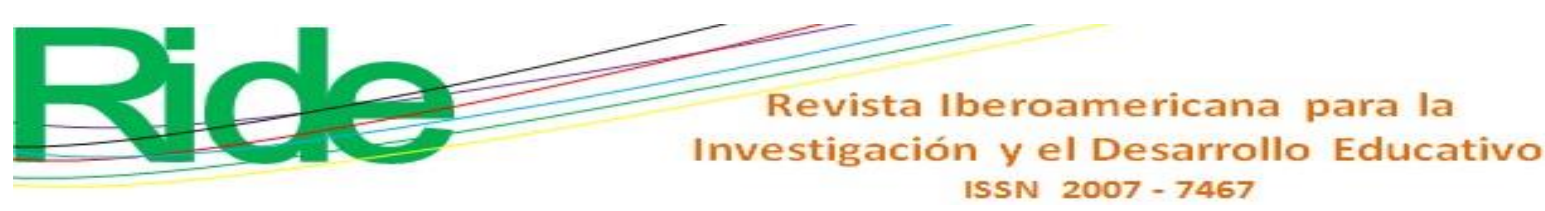

3. Transversalización curricular del eje medio ambiente:

a) Con base en la valoración de los resultados del instrumento 1, se aplica a los docentes del programa educativo el instrumento 2 para que propongan elementos del eje ambiental, viables de incorporarse en la competencia de su unidad de aprendizaje.

b) Se adecua el perfil de egreso con la incorporación de conceptos medioambientales. El comité asesora a los docentes para que revisen las competencias y programa de unidad e incorporen los componentes del eje transversal, cuando sea viable y pertinente.

c) La suma de todos los componentes proporciona una visión global del currículo. Lo ideal es que los elementos del eje estén presentes en un rango entre $76 \%-100 \%$ de las unidades de aprendizaje en cada etapa de formación. Es conveniente que haya una gradualidad en la inclusión de cada componente en las unidades de aprendizaje por etapa de formación; esto significa una presencia de los conocimientos en la etapa de formación institucional; habilidades en la etapa de formación profesional; y actitudes y valores en la etapa de integración y vinculación, sin que esta orientación se convierta en una condicionante.

4. Elaboración de la secuencia didáctica con la transversalización del medio ambiente:

a) Una vez que el eje medio ambiente está transversalizado en el perfil de egreso y en las competencias de las unidades de aprendizaje, el grupo de expertos y el CDC diseñan una propuesta de acompañamiento a toda la planta docente para que "impregnen" su secuencia didáctica.

b) Concluidos los trabajos, el CDC se mantiene mediante un plan de mejora para una evaluación constante del funcionamiento del programa educativo, así como la capacitación y evaluación continua de la planta docente.

\section{Discusión}

Los resultados relevantes pueden sintetizarse en tres ideas: identificación de las necesidades de capacitación docente, estrategias para la transversalización y la propuesta para iniciar un nuevo proceso de investigación.

Se encontró que los docentes que integran los CDC requieren participar en procesos de capacitación y actualización en temas de ambiente, transversalidad y diseño curricular, para un mejor entendimiento conceptual y encabezar la tarea de la transversalización. Hay estudios que manifiestan una necesidad similar de formación docente, como el del Centro de Gestión 


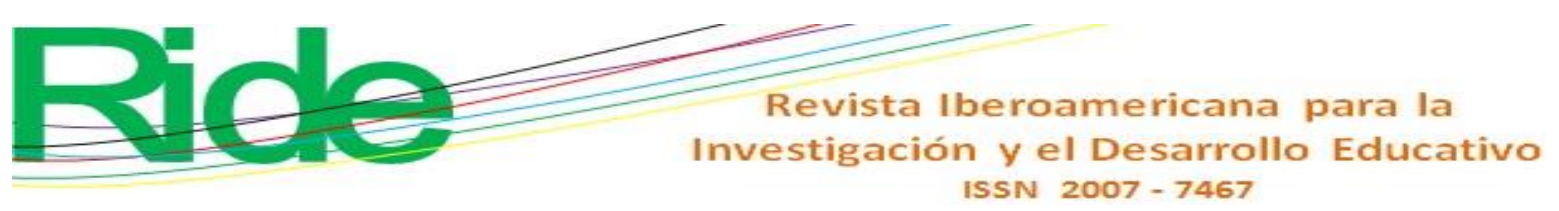

Ambiental y Ecología [Cegae] (2017), Clavijo (2018) y Mora (2012). Cabe referirse al trabajo de Aznar et al. (2018) en la Universidad de Valencia, España (2016-2017), para incluir la sustentabilidad como dimensión transversal en el currículo, involucrando a los docentes de la Maestría en Enseñanza Secundaria. Estos autores indicaron que la sustentabilidad y la educación para el desarrollo sustentable ya habían sido integrados; sin embargo, los programas de formación para los docentes no consideraron metodologías de transposición didáctica para abordar en clase los problemas socioambientales. Concluyeron en la necesidad de fortalecer el aprendizaje de contenidos, procedimientos y actitudes de sustentabilidad para implementarlos en el aula, y que los procesos de capacitación docente son la estrategia más adecuada.

La segunda premisa es que hasta el término del segundo ciclo no se había logrado llegar a la transversalización del eje medio ambiente. Sin embargo, Piza et al. (2018), en una investigación análoga a la que se halla en este documento, identificaron las necesidades de los docentes sobre los temas medio ambiente, transversalidad y competencias docentes. En conjunto con un comité de expertos, se diseñó el curso-taller denominado Transversalidad curricular del eje medio ambiente, con la participación de 24 docentes de la Facultad de Contaduría y Administración de la UAGro; concluyó con la elaboración del programa de unidad de aprendizaje Mercadotecnia III. Este aporte es significativo y será esencial integrarlo a una tercera intervención programada.

Finalmente, se plantea un tercer ciclo de investigación, dividido en cuatro etapas: conformación del CDC, diagnóstico de la transversalidad, transversalización curricular del eje medio ambiente, y la transversalización de la secuencia didáctica o plan de clase. Algunas investigaciones similares también proponen una sistematización de los trabajos. En 2005, la Universidad Nacional del Nordeste, en Argentina, desarrolló una etapa piloto; se interesó en un proceso de ambientalización curricular, con el propósito de reorientar con una conciencia ambiental, social y participativa los perfiles de egreso de los planes de estudios de las Facultades de Medicina (Medicina, Enfermería y Kinesiología), e Ingeniería (Electromecánica y Civil). El Centro de Gestión Ambiental, dependencia de la universidad, estuvo a cargo del proyecto, el cual consta de seis fases de intervención: diagnóstico, evaluación diagnóstica de las facultades, elaboración de una propuesta, implementación, monitoreo del proyecto de ambientalización y evaluación de la ambientalización curricular; así como una fase participativa y permanente de concientización y sensibilización. Destaca que se han logrado modificaciones curriculares en los objetivos y contenidos temáticos de 13 asignaturas. Esta propuesta es interesante porque plantea 


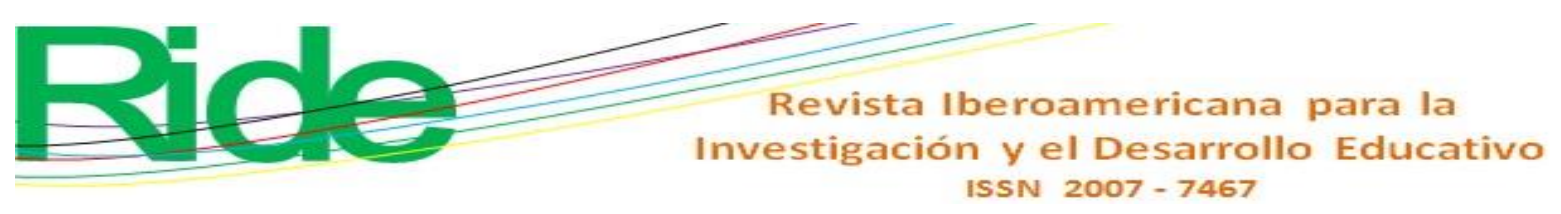

permite la incorporación o adición de elementos ambientales por parte del docente en cada unidad de aprendizaje, según su formación y experiencia profesional.

\section{Conclusiones}

Se subrayan las facilidades institucionales para el desarrollo de esta investigación; destaca la problemática generada por un manejo inadecuado o impreciso de los conceptos tanto curriculares como ambientales - que conllevan a una profunda reflexión sobre el quehacer académico de las universidades, en el ineludible compromiso social con la formación integral de sus estudiantes, futuros tomadores de decisiones.

Aunque el desarrollo de la transversalización ambiental ha sido mínimo, se considera positivo por la participación del docente como principal actor y generador de información. Una limitante de la investigación es que no participaron los mismos CDC en el primero y segundo ciclo, por lo que no se puede precisar el grado de concientización de los 12 participantes del primer ciclo investigativo.

La grave problemática ambiental planetaria que estamos viviendo demanda mayor compromiso de las instituciones de educación superior en la generación de información confiable y pertinente para su debida atención. Se requiere que la administración central de la UAGro promueva procesos de formación continua con temáticas relacionadas con los emergentes sociales; asimismo, que estimule los esfuerzos de los docentes que se capaciten y logren llegar a la transversalización en su secuencia didáctica o plan de clase.

Los resultados obtenidos sobre la experiencia de 15 CDC participantes permite considerar que la constitución interdisciplinaria de estos cuerpos colegiados es pertinente para trabajar la estrategia de la transversalización curricular. La tarea pendiente consiste en que este tipo de trabajos académicos sean considerados en las políticas administrativas de la universidad.

\section{Agradecimientos}

Finalmente, se agradece a los docentes que integran los CDC participantes por sus contribuciones y disposición, especialmente a los programas de Ciencias y Tecnologías de la Información, Contaduría y Administración. 


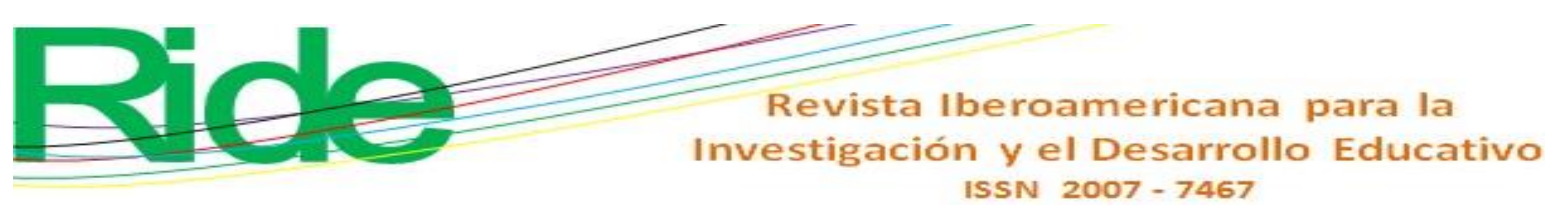

Giannuzzo, N. (2010). Los estudios sobre el ambiente y la ciencia ambiental. ScientiaeStudia, 8(1), 29-156 Recuperado de http://www.scielo.br/scielo.php?pid=S1678$31662010000100006 \&$ script=sci_arttext.

González, E. (2000). Los desafíos de la transversalidad en el currículum en la educación básica en México. Tópicos de Educación Ambiental, 2(6), 63-70. Recuperado de https://dialnet.unirioja.es/servlet/articulo?codigo=203622

González, E. y Arias, M. A. (2015). La investigación en educación ambiental en México: una década a debate. En Castillo, A., Reyes, J. y Castro, E. (coords.), Geometrías para el futuro (pp. 38-60). México: Editorial Universitaria.

Lacre, H. y Mangione, A. (2004). Ambientalización curricular de los estudios superiores, Experiencia piloto en la Universidad Nacional de San Luis. Trabajo presentado en el 1. ${ }^{\circ}$ Congreso de Educación Ambiental para el Desarrollo Sustentable en la República Argentina. Provincia de Córdoba, 6 y 9 de mayo de 2004. Recuperado de http://www0.unsl.edu.ar/ geo/p-geoambiental/geo/docs/produccion/exp_piloto.pdf.

Latorre, A. (2003). La investigación-acción. Conocer y cambiar la práctica educativa (1. ${ }^{a}$ ed.). Barcelona, España: Graó

Lozano, R. (2008). Diffusion of sustainable development in universities' curricula: an empirical example from Cardiff University. Environmental Management for Sustainable Universities, 18(7), 637-644.

Mora, W. M. (2012). Ambientalización Curricular en la Educación Superior: Un estudio cualitativo de las ideas del profesorado. Revista de currículum y formación del profesorado, 16(2), 77-103. Recuperado de https://www.ugr.es/ recfpro/rev162ART6.pdf.

Moreno, T. (2011). Didáctica de la Educación Superior, nuevos desafíos en el siglo XXI. Perspectiva Educacional, Formación de profesores, 50(2), 26-54.

Piza, V., Aparicio, J. L., Rodríguez, C., Marín, R., Beltrán, J. and Bedolla, R. (2018). Sustainability in Higher Education: A Didactic Strategy for Environmental Mainstreaming. Sustainability, 10(12), 1-12. Retrieved from doi.org/10.3390/su10124556.

Programa de las Naciones Unidas para el Medio Ambiente [Pnuma]. (2006). Valoración del daño ambiental. Ciudad de México, México: Programa de las Naciones Unidas para el Medio Ambiente. Recuperado de http://www.pnuma.org/gobernanza/documentos/Valoracion_Dano_Ambiental.pdf. 


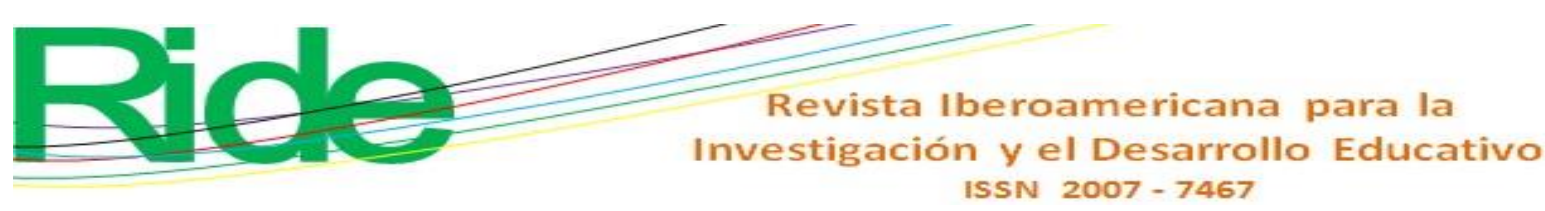

Santamaría, O. y Bravo, M. (2015). Ambientalización curricular de las instituciones de educación superior. Ponencia presentada en el Simposio Sustentabilidad en la Formación Tecnológica. Zacatenco, Ciudad de México, del 27 de mayo al 8 de septiembre de 2015.

Stubbs W. and Cocklin C. (2008). Teaching sustainability to business students: shifting mindsets. International Journal of Sustainability in Higher Education, 9(3), 206-21. Retrieved from doi.org/10.1108/14676370810885844.

Tovar, M. y Sarmiento, P. (2011). El diseño curricular, una responsabilidad compartida. Colombia Médica, 42(4), 508-517. Recuperado de http://www.redalyc.org/articulo.oa?id=28321543012.

Universidad Autónoma de Guerrero. [UAGro]. (2004). Modelo Educativo y Académico de la $U A G$. México: Edición Especial, Gaceta Universitaria.

Universidad Autónoma de Guerrero [UAGro]. (2012). Diseño, Evaluación y Actualización de Planes de Estudio de Licenciatura (en el marco del Modelo Educativo y Académico de la $U A G)$. México: Universidad Autónoma de Guerrero.

Universidad Autónoma de Guertero [UAGro]. (2017). Segundo Informe de Labores. Dr. Javier Saldaña Almazán. Rector (2013-2017). México: Universidad Autónoma de Guerrero. Recuperado de https://www.uagro.mx/hcu/informes/2do_Informe.pdf.

Velásquez, S. y Jairo, A. (2009). La transversalidad como posibilidad curricular desde la educación ambiental. Revista Latinoamericana de Estudios Educativos, 5(2). 29-44. Recuperado de https://www.redalyc.org/pdf/1341/Resumenes/Abstract_134116861003_2.pdf.

Yus, R. (1996) Temas transversales: hacia una nueva escuela (1. ${ }^{a}$ ed.). Barcelona, España: Graó. 


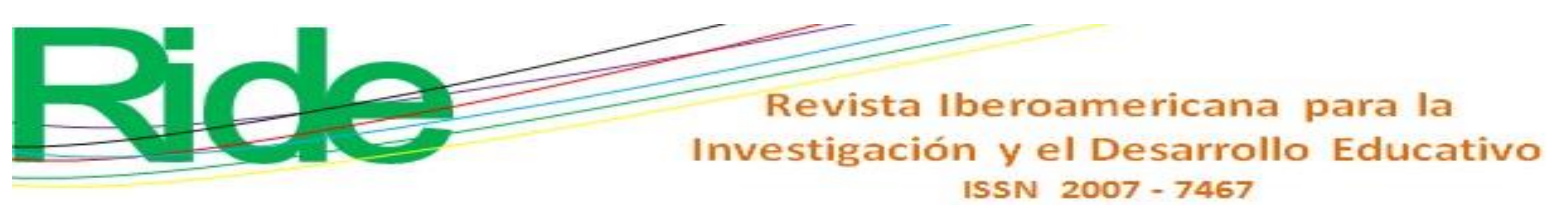

\section{Anexos}

Instrumento 1. Diagnóstico sobre la vinculación del eje transversal medio ambiente en el programa educativo o unidad de aprendizaje con fundamento en el Modelo Educativo de la UAGro MEUAGro

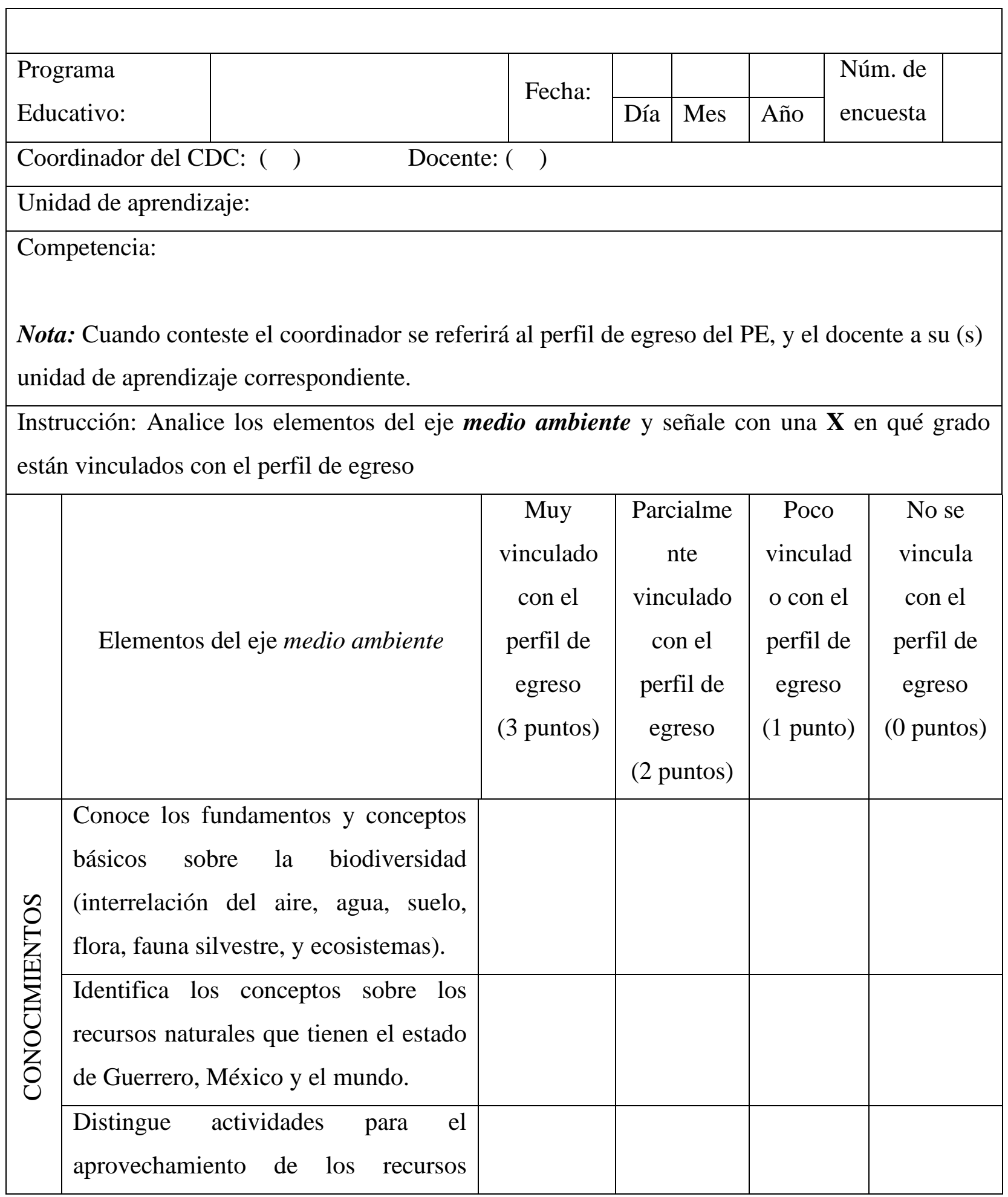




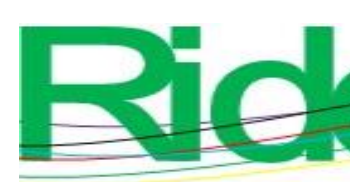

Revista Iberoamericana para la Investigación y el Desarrollo Educativo

ISSN 2007 - 7467

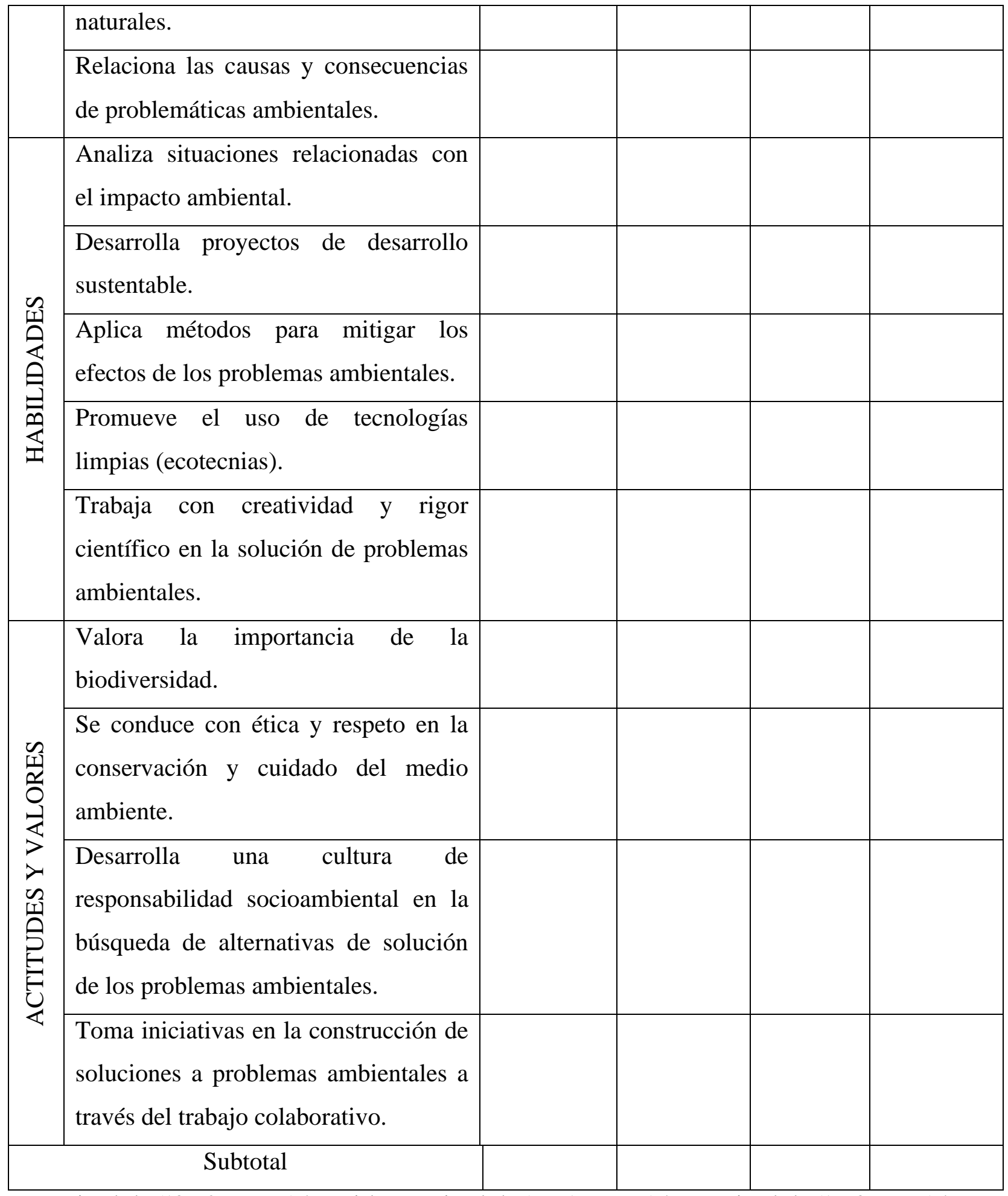

Muy vinculado (18 - 26 puntos) | Parcialmente vinculado (9 - 17 puntos) | Poco vinculado (1 - 8 puntos) | No se vincula (0 puntos)

Fuente: Elaboración propia con base en Aparicio et al. (2014) 
Instrumento 2. Propuesta del docente para la transversalización del eje medio ambiente en su unidad de aprendizaje

\begin{tabular}{|c|c|c|c|c|c|c|}
\hline \multirow{2}{*}{\multicolumn{2}{|c|}{$\begin{array}{l}\text { Programa } \\
\text { Educativo: }\end{array}$}} & \multirow{2}{*}{ Fecha: } & & & & \multirow{2}{*}{$\begin{array}{l}\text { Núm. de } \\
\text { entrevista }\end{array}$} \\
\hline & & & Día & Mes & Año & \\
\hline \multicolumn{7}{|c|}{ Unidad de aprendizaje: } \\
\hline \multicolumn{7}{|c|}{ Etapa de formación: } \\
\hline \multicolumn{7}{|c|}{ Competencia: } \\
\hline \multicolumn{7}{|c|}{ Nombre del docente: } \\
\hline \multicolumn{7}{|c|}{$\begin{array}{l}\text { Instrucción: Analice los elementos del eje medio ambiente; si tiene una propuesta o sugerencia } \\
\text { adicional, viable de incorporar en su unidad de aprendizaje, favor de escribirla en la columna } \\
\text { derecha. }\end{array}$} \\
\hline & $\begin{array}{c}\text { Elementos propuestos del eje } \\
\text { medio ambiente }\end{array}$ & & Prof & uesta & l doc & \\
\hline \multirow{4}{*}{ 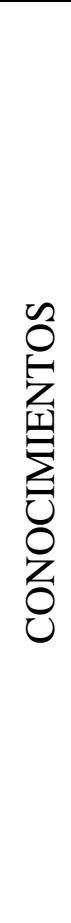 } & $\begin{array}{l}\text { Conoce los fundamentos y conceptos } \\
\text { básicos sobre la biodiversidad } \\
\text { (interrelación del aire, agua, suelo, } \\
\text { flora, fauna silvestre y ecosistemas). }\end{array}$ & & & & & \\
\hline & $\begin{array}{l}\text { Identifica los conceptos sobre los } \\
\text { recursos naturales que tienen el } \\
\text { estado de Guerrero, México y el } \\
\text { mundo. }\end{array}$ & & & & & \\
\hline & $\begin{array}{l}\text { Distingue actividades para el } \\
\text { aprovechamiento de los recursos } \\
\text { naturales. }\end{array}$ & & & & & \\
\hline & $\begin{array}{l}\text { Relaciona las causas y consecuencias } \\
\text { de problemáticas ambientales. }\end{array}$ & & & & & \\
\hline - & Analiza situaciones relacionadas con & & & & & \\
\hline
\end{tabular}


